\title{
ART implantation failure and miscarriage in patients with elevated intracellular cytokine ratios: response to immune support therapy
}

Conor Harrity ${ }^{1}$ (D) Lyuda Shkrobot ${ }^{2}$, David Walsh ${ }^{2}$ and Kevin Marron ${ }^{2^{*}}$ (D)

\begin{abstract}
Background: The origins of adverse reproductive outcome can be multifactorial, but the contribution of the maternal immune system is considered debatable. Elevated intracellular cytokine ratios have been proposed, although not universally supported, as a marker for immunological dysfunction in implantation and early pregnancy. Poor patient selection or inadequate treatment or testing may be confounding factors. Specific immunomodulation, in carefully selected sub-populations of ART patients with poor reproductive history, despite transfer of good quality blastocysts, may potentially improve clinical outcomes.
\end{abstract}

Methods: Intracellular cytokine ratios (CKR) were prospectively assessed in 337 patients presenting with a history of implantation failure and/or pregnancy loss, prior to further treatment, and were found to be elevated in 150 (44. 5\%). Of this group, 134 agreed to initiate a standardised immunotherapy regime (nutraceuticals, prednisolone \& intralipids) to evaluate the efficacy of this proposed therapy. Of the intervention population, a small cohort $(n=70)$ delayed commencing ART for $\sim 10$ weeks to assess if extended pre-treatment nutraceutical supplementation could normalise CKRs prior to starting ART, and if this conferred additional benefit.

Results: Baseline assessment in the intervention population $(n=134)$ identified 160 miscarriages from 180 total pregnancies (89\% miscarriage rate, MR), conceived both spontaneously and by assisted reproduction. Posttreatment analysis of subsequent ART cycles revealed a significant improvement in both implantation (OR 3.0, 2.0-4. $5)$ and miscarriage rates $(41 / 97,42.2 \% M R, P<0.001)$. Interestingly, pre-treatment normalisation of CKRs appeared to impart marginal extra benefit prior to subsequent fertility treatment with immunotherapy.

Conclusions: Following immunomodulation, significant improvements in both implantation rate and miscarriage rate were seen in this poor prognosis population. This suggests a possible role for both detailed immunoevaluation of patients with poor reproductive history with good embryo quality, and application of personalised immunotherapy regimes alongside ART in selected cases. Future randomised controlled trials are needed to definitively evaluate this potentially promising therapeutic approach.

Keywords: Immunomodulation, Cytokines, Implantation, Miscarriage

\footnotetext{
* Correspondence: kevin.marron@sims.ie

${ }^{2}$ Sims IVF Clinic, Clonskeagh Road, Clonskeagh, Dublin 14, Ireland

Full list of author information is available at the end of the article
}

(c) The Author(s). 2018 Open Access This article is distributed under the terms of the Creative Commons Attribution 4.0 International License (http://creativecommons.org/licenses/by/4.0/) which permits unrestricted use, distribution, and reproduction in any medium, provided you give appropriate credit to the original author(s) and the source, provide a link to the Creative Commons license, and indicate if changes were made. The Creative Commons Public Domain Dedication waiver (http://creativecommons.org/publicdomain/zero/1.0/) applies to the data made available in this article, unless otherwise stated. 


\section{Background}

Maternal immunological modulation in pregnancy is a well-established concept [1-4], but interpretation of variations in the immune status of those attempting to conceive but appearing to have implantation failure of good quality embryos, or with recurrent pregnancy loss, is controversial [5-7] Many factors are accepted as necessary for the successful completion of an assisted reproductive technology (ART) cycle from implantation to birth. Oocyte and sperm quality along with the endometrial environment are a few examples of the hurdles which must be cleared early in the process, while maternal allograft suppression and thrombotic risk must be achieved later [8]. The active and particularly innate immune systems are accepted components of this process and many studies link various immunological abnormalities with recurrent implantation failure (RIF) and recurrent pregnancy loss (RPL) [9-12]. Auto-immune factors such as anti-phospholipid antibody syndrome and anti-thyroid peroxidase antibodies have also been shown to influence pregnancy outcome [13-16] and empirical treatment in these cases is recommended [17]. Although not standard practice, testing for immunological abnormalities in the lymphocyte populations specifically, is gaining more attention in this field. Circulating $\mathrm{T}$ cells, cytokine expression bias and natural killer cells are known to be fundamental to a successful pregnancy [18] so in this regard, testing of this nature seems justified. NK cytotoxicity, for example, has been reported as being predictive of RPL [19]. Glucocorticoid steroids have long been the agents of choice in attempts to influence the expression and activity of pro-inflammatory mediators and have been used as a key component of many proposed immunomodulatory regimens [15, 20-22]. Similarly, IVIG and intralipid infusions have been used as adjunctive agents to both supress and confuse the maternal adaptive immune system around the window of implantation [23]. Intralipid has been proposed to exert a modulating effect on certain immune cellular mechanisms, potentially down-regulating cytotoxic or activated natural killer cells (NKa) [24]. This effect may act synergistically with the concomitant administration of corticosteroids, such as dexamethasone or prednisolone, to suppress cytotoxic/activated T-lymphocytes. A potential net effect of combined intralipid and corticosteroid therapy might be the ability to suppress pro-inflammatory cellular (Type-1) cytokines such as interferon gamma and TNF-alpha. Indeed, in-vitro testing has shown that Intravenous Intralipid administration can successfully downregulate natural killer cell activation $(\mathrm{NKa})$ within $2-3$ weeks in up to $78 \%$ of women experiencing suspected immunologic implantation dysfunction [25-27].

The capacity to produce monomeric cytokines, either pro-inflammatory, (TNF- $\alpha$, IFN- $\gamma$ ) or anti-inflammatory (IL-10), exists in many lymphocyte subsets [28]. Stimulated intracellular cytokine expression levels in peripheral blood lymphocytes, specifically CD4+ T cells, has shown an association between the levels of expression of these markers and the incidence of both recurrent spontaneous abortions and recurrent implantation failure [29]. Intracellular cytokines of this nature are known to stimulate activatory KIR receptors on NK cells and thus render them more cytotoxic [30]. Pharmaceuticals such as Humira (adalimumab) and Enbrel $^{\circledR}$ (etanercept) are sometimes used in the miscarriage/implantation failure patient who presents with raised intracellular cytokines [31], but not without controversy due to their toxicity profile and the lack of general acceptance for requirement of this course of action. The immunomodulatory regime employed in this study is an attempt to provide a similar physiological response, an improved outcome and a reduced risk of adverse effects.

There is some evidence that complementary treatment with appropriate supplements and anti-oxidants, such as Vitamin D3, can also improve the natural conception rate of sub-fertile couples, and increase the success rate of assisted reproductive techniques [32]. Vitamin D is a well-established immune modulator and may have an influence on reproductive capacity, with deficiency associated with autoimmune problems [33] and recurrent pregnancy loss (RPL) [34]. Vitamin D has many functions but in the reproductive setting it is thought to regulate $\mathrm{T}$ helper cells and decrease Th1 response while promoting suppressor Th2 cells, thus helping the body maintain a pregnancy [35]. Omega 3 fish oils, especially Eicosapentaenoic acid (EPA), have been shown to potentially decrease NK cell activity by up to $48 \%$ [36]. It may also inhibit in vivo TNFa by up to 74\% [37], with cytokine production decreasing as EPA levels rise. The mediators of all these effects are thought to be modifications to the production and release of pro-inflammatory cytokines such as TNFa and IFNg, which are primarily the purview of CD4+ T cells and NK cells. These studies led us to hypothesise that treatment with glucocorticoids and intralipid infusions, combined with Vitamin D3, and omega 3 fatty acids in conjunction with B complex vitamins may have a positive immunomodulatory effect, and benefit some patients in achieving and maintaining pregnancy. By focusing our observations on selected RPL/ RIF subpopulations, where potential immune derangement is more likely, especially with baseline cytokine ratio elevation, specific and appropriate patient subgroups may be identified where immunomodulation can be applied to best effect.

\section{Methods}

The study design was a longitudinal prospective cohort comprising a full historical evaluation of patient demographics and outcomes at presentation, followed by intracellular cytokine testing for inclusion, if appropriate, 
and subsequent treatment with additional immunotherapy in those with abnormal baseline levels. Patients presenting with a history of early pregnancy losses or failed embryo transfers were offered an initial assessment of their CD4+ intracellular cytokine ratios (CKR) prior to their next ART cycle using the investigative technique as previously described [38]. Enrolment took place in a single private university affiliated ART centre over a 3 year period, from March 2013 to March 2016. Inclusion criteria for offering this test was a background of either $>2$ miscarriages or minimum 2 unsuccessful transfers of good quality embryos, with female age $<42$ years. An anatomically normal endometrial cavity on saline infusion sonogram and normal endocrine profile (thyroid function and prolactin within normal ranges) were also required. Normal ranges for interpreting CKR's were based on levels established originally by Rosalind Franklin Labs, Chicago, and are reported as 13.2-30.6 for TNF- $\alpha$ : IL-10 and 5.8-20.5 for IFN- $\gamma$ : IL-10 [29]. Levels above these established ratios are considered "High". We also report the individual percentage expression levels which constitute the ratios. Freshly collected whole blood cells were diluted into Iscoves modified Dulbecco's medium (IMDM, Gibco Life technologies) fortified with 5000 IU penicillin \& streptomycin (Gibco life technologies) and separated into stimulated and un-stimulated groups. The stimulated aliquot had $50 \mathrm{ng} / \mathrm{ml}$ phorbol myristate acetate (PMA) and $1 \mathrm{micromol} / \mathrm{L}$ ionomycin added in the presence of $1 \mathrm{uL}$ Golgi Plug protein transport inhibitor (Sigma Aldrich, UK) to allow for protein accumulation in the Golgi complex. The unstimulated group had only the Golgi Plug introduced. Incubation took place overnight for $\sim 18 \mathrm{~h}$ at $37^{\circ}$ centigrade in $5 \%$ $\mathrm{CO} 2$. All cells were then processed and stained according to the manufacturer's instruction with the $\mathrm{BD}$ whole blood Cytotoxic/Cytoperm kit (BD Pharmingen). Analysis on the Navios ${ }^{\mathrm{Tm}} 10$ colour 3 laser flow cytometer (Beckman Coulter UK) then followed immediately using dedicated software. Post incubation, the cells were lysed in Pharmlyse buffer and following washing and centrifugation steps, were incubated with antibodies to surface ligands CD45 (anti-human Krome Orange, clone J.33), CD3 (anti-human Pacific Blue clone UCHT1) and CD8 (anti-human PC7 Clone B9.11), followed by a fixation and permeabilisation step. To detect intracellular cytokines, monoclonal antibodies to TNF- $\alpha$, IFN- $\gamma$ and IL-10 were employed, specifically phycoerythrin (PE)-anti-human TNF- $\alpha$ clone IPM2, Fluorescein isothiocynate (FITC)- anti- human IFN- $\gamma$ clone 45.15 (both Beckman Coulter UK) and allophycocyanin (APC)-anti human IL-10 clone JES3-19F1 (BD Pharmingen). Corresponding isotype controls were utilized for each antibody and for each patient as well as fluorescence minus one (FMO) controls.
Included patients were stratified into subgroups. Recurrent pregnancy loss was defined based recent on ESHRE guidelines of 2 or more miscarriages prior to 22 weeks [39]. Ultrasonic evidence, in addition to positive hCG, denoted clinical pregnancy [40]. As the RPL population is a heterogeneous group encompassing many aetiologies, we proposed a further subdivision into patients with non-consecutive miscarriages (RM), two consecutive miscarriages (RM2) and those with more (RM3+) to determine if differences were present. Implantation failure (IF) and repeated implantation failure (RIF) are concepts unique to assisted reproduction, with many factors important in the assignment of this terminology, including early bhCG evaluation, number of embryos transferred and number of previous IVF failures [41]. RIF for the purposes of this study was categorised as a background of primary infertility, combined with the published definition of a minimum of two unsuccessful blastocyst transfers following at least two attempts [42]. Despite these entry criteria there does exist a third population which cannot be clearly assigned to either the RPL or RIF group and these have been denoted as "Other". These patients presented with secondary infertility and a either a mixed combination of IF and RPL, or new onset implantation failure after previous live birth.

A total of 337 patients were recruited for cytokine analysis, of which $44.5 \%(n=150)$ had ratios above the described normal range, and so were eligible for inclusion in the study (Fig. 1). Enrolment was confirmed after detailed discussion of the potential risks and benefits of using the interventional therapy, with 16 patients declining to take part. The treatment arm was commenced on an immunomodulatory regime of prednisolone $(15-25 \mathrm{mg}$ started with ovarian stimulation or estrogenic endometrial preparation), continuing until 12 weeks of gestation or negative test. Intravenous intralipid infusions (Fresenius Kabi, $20 \% \mathrm{w} / v$, administered biweekly-1 week pre- and 1 week post-embryo transfer, continuing with a final third dose after confirmation of pregnancy). An immune influencing nutritional regimen (Omega $33 \mathrm{~g}$, B complex, Vitamin D3 $25 \mu \mathrm{g} / 1000 \mathrm{iu}$, daily) was used in a subset of patients willing to delay empiric therapy for approximately 10 weeks. Responses to this approach are previously described [38]. Addition of low molecular weight heparin (Enoxaparin/ Clexane 20-40 mg SC as per BMI) was utilised, if appropriate, based on thromboelastogram result (INTEM-s ${ }^{\circ}$ MCF result $\geq 64 \mathrm{~mm}$ ) [43]. Controlled ovarian stimulation was performed using long agonist (Buserilin ${ }^{\mathrm{Tm}}$ ) or short antagonist (Cetrotide Merck) protocols, with either recombinant FSH (Gonal-F $\mathrm{F}^{\mathrm{m}}$, Merck UK Ltd) or hMCG (Menopur $^{\mathrm{rm}}$, Ferring LTD) based on age, AMH, baseline gonadotropin levels and antral follicles count. Final oocyte maturation trigger (Pregnyl ${ }^{\mathrm{Tm}}$, MSD or Oviterelle ${ }^{\mathrm{Tm}}$, Merck UK LTD) was administered once $\geq 2$ follicles were $\geq$ 


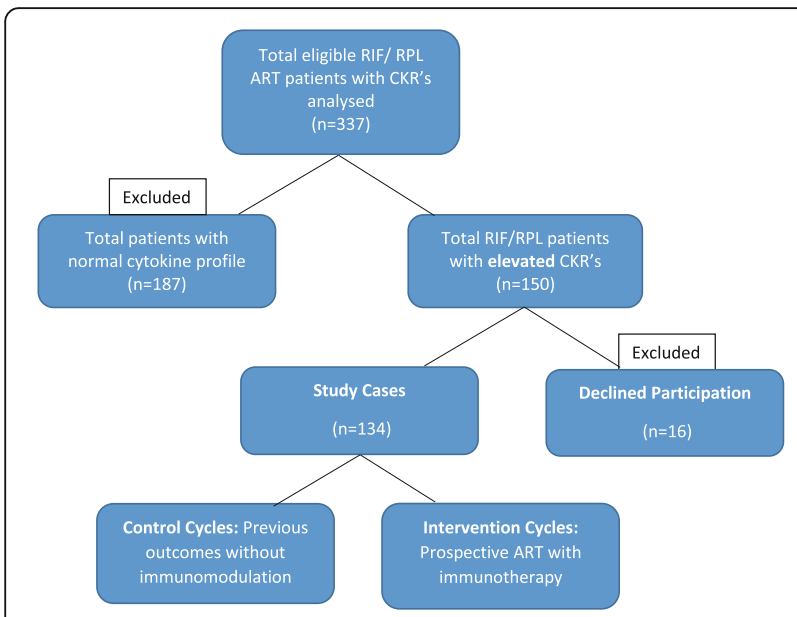

Fig. 1 Flow chart representing patient enrolment. Only those patients who met the entry criteria AND presented with elevated CKR's were enrolled in the study. The entry criteria for CKR assessment were a history of RIF and/or RPL. All participants received standard $A R T$ techniques depending on age, $A M H, B M l$ etc. ART; assisted reproductive techniques, RIF; repeat implantation failure, RPL; recurrent pregnancy loss

$17 \mathrm{~mm}$, and progestogenic luteal phase support was added $\left(\right.$ Crinone $^{\mathrm{TM}} /$ Gestone $^{\mathrm{TM}}$ ) and continued until negative pregnancy test, or 12 weeks gestation. Frozen embryo transfer cycles were performed using a HRT protocol without pituitary downregulation with transfer at $\mathrm{P}+5$. All donor oocyte treatments were performed following this approach. Clinic practice was to recommend routine extended embryo culture to blastocyst for transfer. PGT-A was not available in the centre during the study period, so not performed on either control or interventional cycles.

A small group of patients $(n=16)$, declined to take part in the study after full counselling despite having elevated cytokine ratios, and proceeded directly to ART as per standard unit protocols, without the addition of immunosuppressive therapy. Control cycles were defined as those ART cycles occurring historically prior to initial CKR analysis where no immunotherapy was used, with both internal clinic data and external centres included, along with spontaneously conceived pregnancies. The interventional arm comprised the subsequent cycles in the same patients, following the CKR evaluation and using the empirical immunotherapy regime described above. Prospective observation was performed on the interventional cycles, with analysis of subsequent pregnancy and miscarriage rates (Table 1). Informed consent was obtained from patients for the analysis and study approval was obtained from the Clinic's Institutional review board.

Statistical analysis was performed using SPSS v 24.0 to identify if any significant differences were noted. Cytokine data was determined to not be normally distributed so non-parametric analysis by Kruskal Wallis testing was utilised to compare levels between patient subgroups.
Table 1 Baseline demographics

\begin{tabular}{lll}
\hline Patient demographics & Treatment Arm & Excluded Cases \\
\hline Age (Mean \pm SD) & $37.8 \pm 4.7$ & $37.7 \pm 6.1$ \\
Total patients (n) & 134 & 16 \\
Classification (n(\%)) & & \\
Repeat Implantation failure (RIF) & $56(41.0)$ & $6(37.5)$ \\
Recurrent Pregnancy Loss (RPL) & $46(35.1)$ & $6(37.5)$ \\
Other & $32(23.9)$ & $4(25.0)$ \\
Pre-intervention obstetric history & & \\
Total pregnancies & 180 & 3 \\
Miscarriages & 160 & 21 \\
Miscarriage rate & $88.9 \%$ & 2 \\
Post-intervention outcomes & & $100 \%$ \\
Total pregnancies & 103 & 2 \\
Miscarriages & 44 & $42.7 \%$
\end{tabular}

Baseline demographics in patients with elevated CKR's $(n=150)$. Patients treated with immunotherapy in addition to standard ART comprise the study population $(n=134)$ while those who declined immunomodulation $(n=16)$ were excluded

Chi square analysis was used to compare proportions, with McNemar's test applied as a repeated measures method to account for multiple pregnancies for individual patients when appropriate. Odds ratios were used to assess the strengths of associations. As there is no central birth register for ART pregnancies in Ireland, ongoing pregnancy at 12 weeks' gestation was chosen to be the primary outcome measure. Patients were referred on to regional maternity units after this stage making follow up more difficult. The majority of miscarriages, however, are expected to occur in the first trimester, with later losses considered less likely.

\section{Results}

During the study time period 337 eligible patients had CKRs assessed, and were found to be elevated in 150 (44.5\%). After medical counselling 134/150 elected to use the study therapeutic regime, 16 declined the addition of immunomodulation and study participation and so were excluded from the analysis. PGT-A was not utilised in any cases. There were no significant differences in demographics between patients joining the intervention group and the excluded cases, so no bias was introduced when these patients elected not to procced in the study (Table 1 ). None of the included patients tested true positive for antiphospholipid antibodies, specifically anti-cardiolipin IgG and anti b2 glycoprotein 1 . There were no Mullerian tract abnormalities found on initial endometrial cavity assessment. Potential coagulation disorders were assessed using maximal clot formation (MCF) via thromboelastogram (ROTEM ${ }^{\mathrm{TM}}$, Werfen UK LTD), with the threshold for 
miscarriage risk as described by Rai et al. [43], and treatment with low molecular weight heparin (Enoxaparin/ Clexane 20-40 mg as per BMI) added if appropriate. The intervention group consisted of 47 (35\%) patients with a classical history of recurrent pregnancy loss, while 55 (41\%) met the criteria for repeated implantation failure. Thirty-two patients (24\%) met the overall study inclusion criteria but could not be clearly classified into either RPL or RIF groups and are deemed "other". Baseline assessment of the studied population $(n=134)$ showed elevated CKRs across the treated groups irrespective of clinical presentation (Table 2). There was no difference in TNFa:IL10 or IFNg:IL10 ratios for the RIF and RPL groups, however RPL patients demonstrated significantly higher percentage expression of TNFa $(P=0.036)$. Regarding coagulation status there was also no significant difference, with the RPL group having a mean MCF of 65.2 (range 62-75), compared to 64.7 (range 62-70) for the RIF patients.

Prior to immunotherapy initiation, historical assessment of the intervention group $(n=134)$ identified 160 previous miscarriages from 180 total pregnancies, and an extremely high miscarriage: pregnancy ratio (MR) of $89 \%$ (Table 1). Subsequent analysis of the post-treatment cycle outcomes in the treated population revealed that 103 pregnancies were achieved, with 44 ending in miscarriage, resulting in a MR of $42.7 \%(P<0.001$, Table 3$)$. There was no difference in MR between the RIF and RPL groups after treatment $(p=0.97)$. When the RPL group was subdivided, there was no difference in either outcomes or baseline cytokine levels between those with 2 consecutive miscarriages or those $\geq 3$. Due to the very small group sample size $(N=2)$, it was not possible to conclude if those with non-consecutive miscarriages form a distinct population or not.

Interestingly, implantation rates for the entire cohort demonstrated significant improvements, with an Odds

Table 2 CKR expression across patient aetiology

\begin{tabular}{llllll}
\hline Group & \multicolumn{5}{l}{ Cytokine expression: M $(\bar{x})$} \\
\cline { 2 - 6 } & TNFa:IL10 & IFNg:IL10 & IL-10 & TNFa & IFNg \\
& $($ ratio $)$ & (ratio) & $(\%)$ & $(\%)$ & $(\%)$ \\
\hline RIF & $63.6(54.0)$ & $16.7(21.8)$ & $0.80(0.87)$ & $44.9(49.8)$ & $15.3(17.3)$ \\
RPL & $54.8(62.6)$ & $20.1(23.0)$ & $0.95(0.93)$ & $55.2(53.2)$ & $18.2(21.2)$ \\
Other & $68.4(72.0)$ & $21.8(23.4)$ & $0.60(0.71)$ & $42.2(48.4)$ & $13.7(15.6)$ \\
Sig. (P) & 0.086 & 0.470 & $0.027^{*}$ & $0.036^{*}$ & 0.315 \\
\hline
\end{tabular}

Mean baseline intracellular cytokine ratios and percentage expression in the study group $(n=134)$, stratified by patient aetiology. ANOVA demonstrates no significant difference in baseline stimulated intracellular cytokine expression or ratios between the populations. Only patients with elevated ratios met study inclusion criteria. RIF patients presented with repeated implantation failure (no pregnancy following at least 2 transfers of good quality embryos). RPL are patients with recurrent pregnancy loss. "Other" refers to secondary infertility patients with features of RIF/RPL, not meeting the strict criteria for either group. *indicates statistical significance to $P<0.05$
Ratio of 3.0 (95\% CI 2.0-4.5) for successful implantation following immunotherapy treatment in those with high baseline cytokine ratios. When stratified by ART type, the differences were most prominent with FET and donor oocyte transfer cycles following immunomodulation (Table 4). Of note, the multiple gestation rate increased despite a reduction in the numbers of embryos being transferred. Pre-treatment the average number of embryos transferred was 1.66, with a twin incidence of $4 \%$. Post immunomodulation the average number of embryos per transfer was lower, reducing to1.38, yet with a higher twin pregnancy rate of $14.7 \%$.

A subgroup of 70/134 patients from the intervention group elected to attempt to normalise their CKR levels with extended nutraceutical supplementation therapy prior to commencing pharmacological immunomodulation and ART, as previously described [38]. At baseline assessment 39 of these patients showed raised TNF $\alpha$ and IFN $\gamma$ ratios (high/high) and 31 showed raised TNF $\alpha$ but normal IFN $\gamma$ ratios (high/norm). Following 10 weeks of pre-treatment, considerable normalisation was achieved, with 43/70 reaching normal or normal/low levels, 12 being partially responsive, and 15 remaining unchanged. Nutraceutical pre-treatment led to an average 6 fold increase in IL-10 expression ( $5.0 \%$ vs $0.8 \%$ ) and a decline in absolute percentage expression of $10.6 \%$ for TNF $\alpha$ and $4.6 \%$ for IFNY ([38]). Interestingly, this subset analysis showed that cytokine ratio normalisation conferred only marginal additional benefit to the change in miscarriage rate following immunomodulation (Fig. 2). All groups showed a $>50 \%$ reduction in miscarriage: pregnancy ratio after standard immunosuppressive therapy, regardless of their change in CKR. Chi square testing showed this was significant in all categories, however when McNemar's test was applied the reduction in MR in patients with unchanged cytokine levels (91.3\% to $44.4 \%)$ was only approaching statistical significance $(P=0.063)$. Odds ratios, however, demonstrate the highest level of improvement in patient's whose cytokine ratios normalised (Table 4).

\section{Discussion}

Reproductive immunology is on the fringe of accepted medical practice in the world of assisted reproduction, and for every advocate there are many naysayers [5]. The presence of a dominant Th1 type phenotype in patients with recurrent miscarriage or implantation failure is a proposed common underlying pathology $[29,30]$. Controversy exists, however, as to the generality of testing in peripheral blood and its relevance to the uterine environment $[6,44,45]$. There are many methods available looking at varied markers in both peripheral blood and endometrium, assessing both percentage expression and cell numbers of lymphocytes [46]. Stimulated intracellular cytokine ratios in peripheral blood, however, 
Table 3 Pre- and post-treatment outcomes stratified by patient aetiology

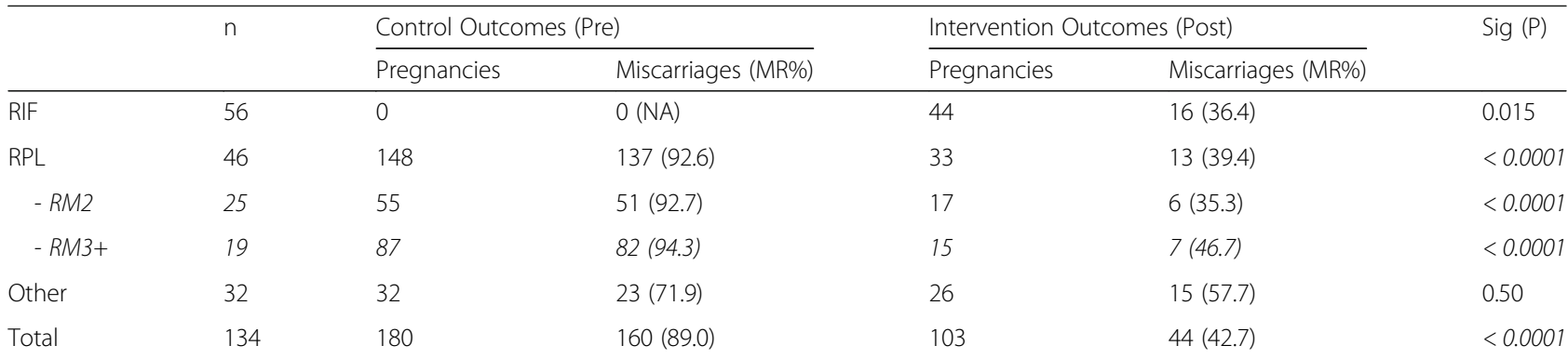

Population outcomes in the study population $(n=134)$ both before (Control outcomes) and after (intervention outcomes) immunomodulation, stratified by patient aetiology. RIF patients presented with repeated implantation failure (no pregnancy following at least 2 transfers of good quality embryos). RPL are patients with recurrent pregnancy loss, the RM2 and RM3+ subgroups refer to either 2 or $\geq 3$ consecutive losses, while the remainder are non-consecutive losses. "Other" refers to secondary infertility patients with features of RIF/RPL, not meeting the strict criteria for either group. Significance calculated using McNemar's test

remains a frequently performed test, but is controversial with many experts not advocating its use. We have previously shown that a dietary regime can be beneficial in significantly reducing intracellular cytokine ratios. Similar TNFa:IL-10 modifications with positive outcomes have been described in other populations [47]. Surprisingly, the movement of intracellular cytokines towards a normal level is not the only influential factor in improving reproductive outcomes, as even the subgroup whose ratios remained unchanged showed a large and significant reduction in miscarriage rates. Rather, it appears that the regime of prednisolone, intralipid infusions and LMWH (if indicated), could be the key influencers. Given the quick transition to a full immunomodulatory regimen, however, it is possible that the effect of the dietary pre-treatment may not yet be fully realised, and a longer preparatory phase may be useful. A broad spectrum immunotherapy regime was devised to tackle potential issues via several possible pathways and mechanisms of action. The aim of this was to maximise the therapeutic benefit of our immune modulatory protocol, however, a detrimental limitation is that it becomes difficult to assess the relevant contribution of each agent and to determine which individual therapy has the most beneficial influence. Further research is required to determine which agents, either alone or together, could potentially have the greatest effect. The ability to determine the relative effect of each component would allow a more personalised protocol for each individual patient. Studies assessing the individual relative benefits of the empiric treatments described here have previously been conducted with variable findings both advocating and not advocating their use [21, 48, 49]. The success of the regimen employed here as evidenced by improved implantation, successful pregnancies and a dramatic reduction in the overall miscarriage rate demonstrates both the combined beneficial effect of these agents as well as the potential importance of specific immunological testing in selected cases. The origin of the underlying difficulty in these patients, be it implantation failure, early versus late miscarriage or consecutive miscarriages, may be very different. Peripheral blood immunophenotyping to an even deeper level, perhaps encompassing KIR receptors, is becoming more achievable, however, it is possible that endometrial biopsy evaluations may be even more helpful in the evaluation and treatment of these difficult cases. The use of TNFa inhibitors such as adalimumab (Humira ${ }^{\oplus}$, AbbVie) may be of use in the populations that either do not respond to, or continue to have an adverse outcomes with this standard regime, but certainly further research on this approach is also needed. A prospective, blinded, randomised controlled trial,

Table 4 Pre- and post-implantation outcomes stratified by treatment modality employed

\begin{tabular}{|c|c|c|c|c|c|c|c|c|c|c|}
\hline & \multicolumn{4}{|c|}{ Control Cycles (Pre) } & \multicolumn{4}{|c|}{ Intervention Cycles (Post) } & \multirow[t]{2}{*}{ OR $(95 \% \mathrm{Cl})$} & \multirow[t]{2}{*}{$\operatorname{Sig}(p)$} \\
\hline & ETs & Embryos & GSs & $\operatorname{IR}(\%)$ & ETs & Embryos & GSS & $\operatorname{IR}(\%)$ & & \\
\hline$\overline{\mathrm{IVF}}$ & 75 & 125 & 25 & 20.0 & 55 & 79 & 22 & 27.8 & $1.54(0.8-3.0)$ & 0.196 \\
\hline ICSI & 48 & 87 & 9 & 10.3 & 69 & 80 & 15 & 18.8 & $2.0(0.8-4.9)$ & 0.127 \\
\hline FET & 30 & 44 & 1 & 2.3 & 67 & 96 & 28 & 29.2 & $17.7(2.3-134.9)$ & 0.006 \\
\hline DE & 24 & 39 & 3 & 7.7 & 60 & 92 & 41 & 44.6 & $9.6(2.8-33.6)$ & 0.0004 \\
\hline Overall & 177 & 295 & 38 & 12.9 & 251 & 347 & 106 & 30.5 & $3.0(2.0-4.5)$ & 0.0001 \\
\hline
\end{tabular}

Implantation rates (IR) in the studied group of 134 patients, before (Pre) and following (Post) immunomodulatory therapy. Significant improvements seen in IR following immunomodulatory therapy in FET and particularly DE cycles. ETs Embryo transfers, GSs Gestational sacs seen, IVF in-vitro fertilisation cycles, ICSI intracytoplasmic sperm injection cycles, FET Frozen embryo transfer cycles, DE Donor egg cycles 


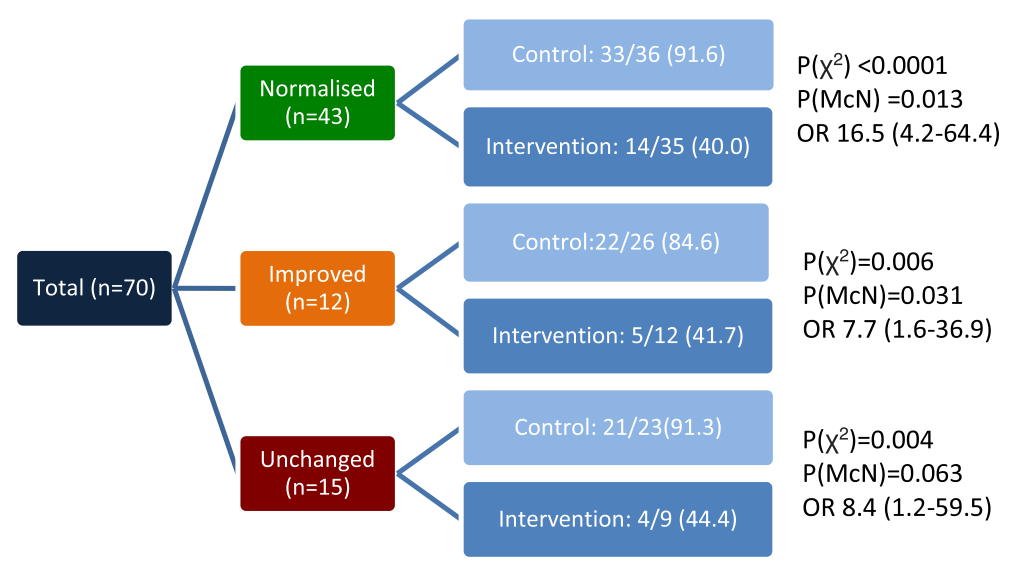

Fig. 2 Cytokine changes and pregnancy outcomes in the patient subset who received nutraceuticals. A cohort of patients (70/134) received a 10 week nutraceutical regime consisting of high dose omega 3, Vitamin D and Vitamin B6 followed by repeated cytokine analysis prior to ART with immunomodulatory intervention (prednisolone and intralipid infusions). This chart illustrates the breakdown of pre- treatment (control) and posttreatment (intervention) pregnancy outcomes for patients who achieved normalisation, improvement or no change in their CKR levels. Significance levels calculated using Chi Square $\left(X^{2}\right)$, McNemar's test $(M c N)$, and Odds Ratio $(\mathrm{OR})$ for reduction in miscarriage following immunomodulation

allocating patients with elevated CKRs to the standardised immunomodulation/ nutraceutical regime, or a placebo, would be the way to definitively confirm the findings of this study.

The data demonstrates that in this high-risk population, a significantly higher implantation and pregnancy rate is achieved in patients empirically prescribed a standardised immunotherapy regime compared to the same patients who previously had similar ART procedures without additional immune supports approximately 2 years previously. A delay of this magnitude would generally be accepted as affording a poorer prognosis to these patients rather than an improved one. The success of the regime in this population may, in part, be ascribed to more discerning immunophenotyping.

\section{Conclusion}

Elevated cytokine ratios have been linked with adverse reproductive outcomes. Proposed treatment protocols have often utilised biological immunomodulators, which antagonise TNFa, but these agents have cost implications, and potentially cytotoxic side effects, particularly with repeated or prolonged exposure. A combination regime with a lower associated risk profile could be more patient friendly, safer, less controversial, and have better compliance. A standardised immunotherapy regime may lead to improved obstetric outcome for a proportion of patients identified to be high risk at baseline testing, reserving the need for more aggressive treatments to those who do not respond to first line interventions, such as those with high order RPL. Unfortunately, the study data is limited by small sample size and lack of randomisation, but the findings are very promising and would suggest that a large prospective randomised controlled trial, to eliminate potential bias and confounding factors, would be useful to determine if this theory is indeed credible. We feel we have identified a population subgroup in which our approach is merited, and with appropriate and timely interventions can allow these patients to realise their strong desire to become parents.

\section{Abbreviations}

ART: Assisted reproductive technologies; CKR: Intracellular cytokine ratios; EPA: Eicosapentaenoic acid; IF: Implantation failure; IFNg: Interferon gamma; IL-10: Interleukin 10; IVF: in-vitro fertilisation; LMWH: Low molecular weight heparin; MR: Miscarriage: Pregnancy Ratio; NK cells: Natural killer cells; RIF: Repeat implantation failure; RM: Recurrent Miscarriage (non-consecutive); RPL: Recurrent pregnancy loss; T cells: T lymphocytes; Th1: T helper 1; Th2: T helper 2; TNFa: Tissue necrosis factor alpha

\section{Acknowledgements}

Thanks to the patients and staff of Sims IVF for their assistance in the work, also sincere thanks to Ms. Gaye Godkin, Clinical Nutritionist, for her input into the design of the nutraceutical regimen employed here and the patients who participated in the study.

Funding

This research was wholly funded by Sims IVF Clinic, Dublin, Ireland.

\section{Availability of data and materials}

The datasets used and/or analysed during the current study are available from the corresponding author on reasonable request.

\section{Authors' contributions}

KM performed all experimental protocols, collated data and co-wrote manuscript. $\mathrm{CH}$ was secondary clinician involved in database design, patient treatment, statistical analysis and co-wrote article. DW Was lead clinician on the design and implementation of the immunomodulation protocol. LS was secondary clinician and assisted with data analysis. All authors read and approved the final manuscript.

\section{Authors' information}

Kevin Marron is Clinical laboratory manager at one of Ireland largest IVF Clinics. He gained his PhD in human cardiac immunocytochemistry from Imperial College London in 1996. Now Lab Manager in Ireland's largest IVF group he has developed a strong research interest in reproductive immunology following up on patients who fail to conceive or lose pregnancies in unusual circumstances. 


\section{Consent for publication}

$\mathrm{N} / \mathrm{A}$

\section{Competing interests}

The authors declare that they have no competing interests. No conflict of interest is declared by any of the authors. Patients all gave informed consent for their data to be used in this study. This research did not receive any specific grant from funding agencies in the public, commercial, or not-for-profit sectors.

\section{Publisher's Note}

Springer Nature remains neutral with regard to jurisdictional claims in published maps and institutional affiliations.

\section{Author details}

${ }^{1}$ Royal College of Surgeons Ireland, 123 St Stephen's Green, Dublin 2, Ireland. ${ }^{2}$ Sims IVF Clinic, Clonskeagh Road, Clonskeagh, Dublin 14, Ireland.

\section{Received: 3 April 2018 Accepted: 27 September 2018}

\section{Published online: 17 October 2018}

\section{References}

1. Sargent I. Maternal and fetal immune responses during pregnancy. Exp Clin Immunogenet. 1992;10(2):85-102.

2. Sacks GP, Redman CW, Sargent IL. Monocytes are primed to produce the Th1 type cytokine IL-12 in normal human pregnancy: an intracellular flow cytometric analysis of peripheral blood mononuclear cells. Clin Exp Immunol. 2003;131(3):490-7.

3. Van Nieuwenhoven AV, Heineman M, Faas M. The immunology of successful pregnancy. Hum Reprod Update. 2003;9(4):347-57.

4. Mor G, Cardenas I. REVIEW ARTICLE: the immune system in pregnancy: a unique complexity. Am J Reprod Immunol. 2010;63(6):425-33.

5. Moffett A, Shreeve N. First do no harm: uterine natural killer (NK) cells in assisted reproduction. Hum Reprod. 2015;30(7):1519-25.

6. Sacks G. Enough! Stop the arguments and get on with the science of natural killer cell testing. Hum Reprod. 2015;30(7):1526-31.

7. Heneghan C, Spencer EA, Bobrovitz N, Collins DR, Nunan D, Pluddemann A, Gbinigie OA, Onakpoya I, O'Sullivan J, Rollinson A, et al. Lack of evidence for interventions offered in UK fertility centres. BMJ (Clinical research ed). 2016; 355:i6295

8. Lee RM, Silver RM. Recurrent pregnancy loss: summary and clinical recommendations. Semin Reprod Med. 2000;18(4):433-40.

9. Beer AE, Kwak JY, Ruiz JE. Immunophenotypic profiles of peripheral blood lymphocytes in women with recurrent pregnancy losses and in infertile women with multiple failed in vitro fertilization cycles. Am J Reprod Immunol. 1996;35(4):376-82.

10. Ntrivalas El, Kwak-Kim JY, Gilman-Sachs A, Chung-Bang H, Ng SC, Beaman KD, Mantouvalos HP, Beer AE. Status of peripheral blood natural killer cells in women with recurrent spontaneous abortions and infertility of unknown aetiology. Hum Reprod. 2001;16(5):855-61.

11. Ghafourian M, Karami N, Khodadadi A, Nikbakht R. Increase of CD69, CD161 and CD94 on NK cells in women with recurrent spontaneous abortion and in vitro fertilization failure. Iran J Immunol. 2014;11(2):84-96.

12. Thum MY, Bhaskaran S, Abdalla HI, Ford B, Sumar N, Shehata H, Bansal AS. An increase in the absolute count of CD56dimCD16+CD69+ NK cells in the peripheral blood is associated with a poorer IVF treatment and pregnancy outcome. Hum Reprod. 2004;19(10):2395-400.

13. Backos M, Rai R, Regan L. Antiphospholipid antibodies and infertility. Hum fertil. 2002:5(1):30-4.

14. Hewell SW, Hammer RH. Antiphospholipid antibodies: a threat throughout pregnancy. J Obstet Gynecol Neonatal Nurs. 1997;26(2):162-8.

15. Rodriguez Garcia JL, Khamashta MA. Clinical advances of interest in the diagnosis and treatment of patients with antiphospholipid syndrome. Revista clinica espanola. 2013;213(2):108-13.

16. Coulam CB, Kaider BD, Kaider AS, Janowicz P, Roussev RG. Antiphospholipid antibodies associated with implantation failure after IVF/ET. J Assist Reprod Genet. 1997; 14(10):603-8

17. RCOG: The use of Antithrombotics in the prevention of recurrent pregnancy loss. In: Online. 2011.

18. Raghupathy R, Makhseed M, Azizieh F, Hassan N, Al-Azemi M, Al-Shamali E. Maternal Th1- and Th2-type reactivity to placental antigens in normal human pregnancy and unexplained recurrent spontaneous abortions. Cell Immunol. 1999:196(2):122-30.

19. Aoki K, Kajiura S, Matsumoto Y, Ogasawara M, Okada S, Yagami Y, Gleicher N. Preconceptional natural-killer-cell activity as a predictor of miscarriage. Lancet. 1995;345(8961):1340-2.

20. Krigstein M, Sacks G. Prednisolone for repeated implantation failure associated with high natural killer cell levels. J Obstet Gynaecol. 2012;32(6): 518-9.

21. Lee KA, Koo JJ, Yoon TK, Do BR, Ko JJ, Cha KY. Immunosuppression by corticosteroid has no effect on the pregnancy rate in routine in-vitro fertilization/embryo transfer patients. Hum Reprod. 1994;9(10):1832-5.

22. Thum MY, Bhaskaran S, Abdalla HI, Ford B, Sumar N, Bansal A. Prednisolone suppresses NK cell cytotoxicity in vitro in women with a history of infertility and elevated NK cell cytotoxicity. Am J Reprod Immunol. 2008;59(3):259-65.

23. Clark DA, Coulam CB, Stricker RB. Is intravenous immunoglobulins (IVIG) efficacious in early pregnancy failure? A critical review and meta-analysis for patients who fail in vitro fertilization and embryo transfer (IVF). J Assist Reprod Genet. 2006;23(1):1-13.

24. Coulam CB, Acacio B. Does immunotherapy for treatment of reproductive failure enhance live births? Am J Reprod Immunol. 2012;67(4):296-304.

25. Clark DA. Immunological factors in pregnancy wastage: fact or fiction. Am J Reprod Immunol. 2008;59(4):277-300.

26. Kwak JY, Kwak FM, Ainbinder SW, Ruiz AM, Beer AE. Elevated peripheral blood natural killer cells are effectively downregulated by immunoglobulin $\mathrm{G}$ infusion in women with recurrent spontaneous abortions. Am J Reprod Immunol. 1996:35(4):363-9.

27. Ruiz JE, Kwak JY, Baum L, Gilman-Sachs A, Beaman KD, Kim YB, Beer AE. Effect of intravenous immunoglobulin $\mathrm{G}$ on natural killer cell cytotoxicity in vitro in women with recurrent spontaneous abortion. J Reprod Immunol. 1996:31(1-2):125-41.

28. Jung T, Schauer U, Heusser C, Neumann C, Rieger C. Detection of intracellular cytokines by flow cytometry. J Immunol Methods. 1993;159(12):197-207.

29. Kwak-Kim JY, Chung-Bang HS, Ng SC, Ntrivalas El, Mangubat CP, Beaman $K D$, Beer AE, Gilman-Sachs A. Increased T helper 1 cytokine responses by circulating $T$ cells are present in women with recurrent pregnancy losses and in infertile women with multiple implantation failures after IVF. Hum Reprod. 2003;18(4):767-73.

30. Szereday L, Varga p, Szekeres-Bartho J. Cytokine production by lymphocytes in pregnancy. Am J Reprod Immunol. 1997;38(6):418-22.

31. Mekinian A, Cohen J, Alijotas-Reig J, Carbillon L, Nicaise-Roland P, Kayem G, Darai E, Fain O, Bornes M. Unexplained recurrent miscarriage and recurrent implantation failure: is there a place for immunomodulation? Am J Reprod. 2016;76(1):8-28

32. Comhaire $F$. The role of food supplementation in the treatment of the infertile couple and for assisted reproduction. Andrologia. 2010;42(5):331-40

33. Cantorna MT, Mahon BD. Mounting evidence for vitamin D as an environmental factor affecting autoimmune disease prevalence. Exp Biol Med. 2004;229(11):1136-42.

34. Ota K, Dambaeva S, Han AR, Beaman K, Gilman-Sachs A, Kwak-Kim J. Vitamin D deficiency may be a risk factor for recurrent pregnancy losses by increasing cellular immunity and autoimmunity. Hum Reprod. 2014:29(2):208-19.

35. Hayes CE, Nashold FE, Spach KM, Pedersen LB. The immunological functions of the vitamin D endocrine system. Cell Mol Biol. 2003;49(2):277-300.

36. Thies F, Nebe-von-Caron G, Powell JR, Yaqoob P, Newsholme EA, Calder PC. Dietary supplementation with eicosapentaenoic acid, but not with other longchain n-3 or n-6 polyunsaturated fatty acids, decreases natural killer cell activity in healthy subjects aged $>55$ y. Am J Clin Nutr. 2001;73(3):539-48.

37. Caughey GE, Mantzioris E, Gibson RA, Cleland LG, James MJ. The effect on human tumor necrosis factor alpha and interleukin 1 beta production of diets enriched in n-3 fatty acids from vegetable oil or fish oil. Am J Clin Nutr. 1996;63(1):116-22.

38. Marron K, Kennedy JF, Harrity C. Anti-oxidant mediated normalisation of raised intracellular cytokines in patients with reproductive failure. Fertil Res Pract. 2018:4(1)

39. ESHRE: <ESHRE RPL Guideline_28112017_FINAL.pdf>. 2017.

40. committee Ap. Definitions of infertility and recurrent pregnancy loss: a committee opinion. Fertil Steril. 2013;99(1):63.

41. Simon A, Laufer N. Assessment and treatment of repeated implantation failure (RIF). J Assist Reprod Genet. 2012;29(11):1227-39. 
42. Polanski LT, Baumgarten MN, Quenby S, Brosens J, Campbell BK, RaineFenning NJ. What exactly do we mean by 'recurrent implantation failure'? A systematic review and opinion. Reprod BioMed Online. 2014;28(4):409-23.

43. Rai R, Tuddenham E, Backos M, Jivraj S, El'Gaddal S, Choy S, Cork B, Regan L. Thromboelastography, whole-blood haemostasis and recurrent miscarriage. Hum Reprod. 2003;18(12):2540-3.

44. Tang AW, Alfirevic Z, Quenby S. Natural killer cells and pregnancy outcomes in women with recurrent miscarriage and infertility: a systematic review. Hum Reprod. 2011;26(8):1971-80.

45. Quenby S, Farquharson R. Uterine natural killer cells, implantation failure and recurrent miscarriage. Reprod BioMed Online. 2006;13(1):24-8.

46. Seshadri S, Sunkara SK. Natural killer cells in female infertility and recurrent miscarriage: a systematic review and meta-analysis. Hum Reprod Update. 2014;20(3):429-38.

47. Winger EE, Reed JL, Ashoush S, El-Toukhy T, Ahuja S, Taranissi M. Degree of TNF-a/IL-10 cytokine elevation correlates with IVF success rates in women undergoing treatment with adalimumab (Humira) and IVIG. Am J Reprod Immunol. 2011;65(6):610-8

48. Polak de Fried E, Blanco L, Lancuba S, Asch RH. Improvement of clinical pregnancy rate and implantation rate of in-vitro fertilization-embryo transfer patients by using methylprednisone. Hum Reprod. 1993;8(3):393-5 .

49. Hviid MM, Macklon N. Immune modulation treatments-where is the evidence? Fertil Steril. 2017;107(6):1284-93.

Ready to submit your research? Choose BMC and benefit from:

- fast, convenient online submission

- thorough peer review by experienced researchers in your field

- rapid publication on acceptance

- support for research data, including large and complex data types

- gold Open Access which fosters wider collaboration and increased citations

- maximum visibility for your research: over $100 \mathrm{M}$ website views per year

At BMC, research is always in progress.

Learn more biomedcentral.com/submissions 